\title{
PLIF Thermometry Based on Measurements of Absolute Concentrations of the OH Radical
}

\author{
By Johannes Heinze ${ }^{1, *}$, Ulrich Meier ${ }^{1}$, Thomas Behrendt ${ }^{1}$, Chris Willert ${ }^{1}$, \\ Klaus-Peter Geigle ${ }^{2}$, Oliver Lammel ${ }^{2}$, and Rainer Lückerath ${ }^{2}$ \\ ${ }^{1}$ Deutsches Zentrum für Luft- und Raumfahrt (DLR), Institute of Propulsion Technology, Linder Höhe, \\ 51147 Köln, Germany \\ 2 Deutsches Zentrum für Luft- und Raumfahrt (DLR), Institute of Combustion Technology, \\ Pfaffenwaldring 38-40, 70569 Stuttgart, Germany
}

Dedicated to Katharina Kohse-Höinghaus on the occasion of her $60^{\text {th }}$ birthday

(Received August 9, 2011; accepted in revised form November 11, 2011)

\section{Laser-induced Fluorescence / Thermometry / Lean Combustion / Gas Turbine}

A method for measurements of planar temperature distributions based on planar laser-induced fluorescence (PLIF) of the $\mathrm{OH}$ radiacal is described. The technique was developed specifically for the application in lean combustion systems, where $\mathrm{OH}$ equilibrium concentrations are largely independent on equivalence ratio and a function of temperature only. It is thus possible to derive a temperature information from measurements of absolute $\mathrm{OH}$ concentration, which can be obtained from a combined PLIF/absorption measurement.

This paper discusses the basics of the method, and describes validation experiments in high pressure laminar premixed flames which were performed to asses its applicability and accuracy. Therefore, we compared our LIF based results with CARS measurements performed in the same flames. Finally, an example for the application in a lean gas turbine model combustor is discussed.

\section{Introduction}

The knowledge of temperatures, their spatial distributions, as well as their fluctuations in space and time, are a key piece of information indispensable for the design and understanding of technical combustion systems. Spectroscopic temperature measurement techniques have received wide interest due to their high spatial and temporal resolution, as well as their non-intrusive nature. Numerous laser-based techniques for temperature measurements in combustion systems have been developed and applied, like CARS (Coherent Anti-Stokes Raman Spectroscopy) [1], Rayleigh scattering [2], Raman scattering [3], or Laser-Induced Fluorescence (LIF) [4], mainly based on the $\mathrm{OH}$ radical [5,6], but also with species like NO [7,8], or tracer species [9] as tempera-

\footnotetext{
* Corresponding author. E-mail: johannes.heinze@dlr.de
} 
ture indicators. All these methods have limitations with respect to their applicability in technical combustion systems, which are characterized by at least one of the following features affecting utilization of spectroscopic methods: Size, pressure, particle load, toxicity, two-phase flow and - frequently disregarded - operating cost.

PLIF-based temperature imaging methods are particularly attractive for the investigation of complex turbulent flame structures, as they provide a large amount of data in a relatively short time.

There are basically two different approaches to derive temperature information from a LIF measurement. One way is to probe the thermal population distribution of molecular states. This method has the advantage that the concentration of the indicator molecule needs not to be known; the only requirement is that the internal degrees of freedom are in thermal equilibrium. The disadvantage is that the relative populations of at least two molecular states need to be probed simultaneously, which requires usually a high experimental expenditure for excitation of two molecular transitions with different wavelengths [4-6]. Even if only a temporally averaged temperature field is desired, subsequent excitation of two lines using the same laser will inevitably lead to a systematic bias towards higher temperature, because of the nonlinear dependence of both state population and the number density of indicator radicals on temperature.

The second method is based on the measurement of a temperature dependent concentration of the indicator molecule. This method, based on $\mathrm{OH}$ radicals as test species, will be discussed in our paper. It has the advantage of considerably less experimental effort and time for setup of the experiment, because it requires just one laser for excitation of a molecular line. The nearly exponential growth of the $\mathrm{OH}$ concentration with respect to temperature enables the high temperature sensitivity of this measurement technique. Its main drawback results from the usually ambiguous dependence of the $\mathrm{OH}$ concentration on both temperature and stoichiometry $\Phi$. This restricts the application to scenarios with lean mixtures, because at sufficiently low equivalence ratios the $\mathrm{OH}$ equilibrium concentrations depend only weakly on $\Phi$ and mainly on temperature. A second requirement is that the $\mathrm{OH}$ concentrations need to be in chemical equilibrium, because high non-equilibrium concentrations at or near flame fronts will feign higher temperatures. The decay rate of chemical superequilibrium increases with pressure; consequently, high pressures are favourable for the application of this method.

In general, the requirements of both lean mixtures and high pressures are hardly ever fulfilled. There are, however, scenarios that allow application of this technique. The Institutes of Propulsion Technology and Combustion Technology of the German Aerospace Center (DLR) are involved in research on gas turbine combustors, for both power generation and aeroengines. In the field of gas turbine combustor research, recent developments pursue lean premixed (LP) approaches for an efficient reduction of nitric oxide production [10]. In such systems, the concentration measurement-based method meets favourable conditions for its application, because ideally, fuel and air have mixed well enough to establish lean mixtures prior to the onset of combustion. Therefore, this paper discusses the technique of temperature measurement based on planar LIF and absorption of $\mathrm{OH}$ radicals as a promising tool primarily for LP gas turbine combustors, but in a wider sense for any combustion systems that meets the requirements for the applicability of this method. 


\section{Description of the method}

The underlying concept of the temperature measurement technique described here is to measure a local OH LIF signal, which can be converted to a local absolute OH density by means of a simultaneous laser absorption measurement [11]. The $\mathrm{OH}$ density, in turn, can be assigned unambiguously to a temperature if the above mentioned requirements are valid, namely, lean mixtures and chemical equilibrium.

Before the method of converting a LIF signal to an absolute $\mathrm{OH}$ concentration by means of an absorption measurement is addressed, the issue of an unambiguous relationship between concentration and temperature, and its implications with respect to the applicability range of the method, is briefly discussed.

In flame front regions (characterized by high reaction rates) the instantaneous $\mathrm{OH}$ concentration can exceed the equilibrium concentration by far; the superequilibrium depends on equivalence ratio, pressure and - in the case of a gas turbine - preheat temperature of the reactants, and is therefore difficult to quantify. In general, the superequilibrium decreases with increasing pressure; as a guideline, for methane/air combustion at 6 bar and an equivalence ratio of 1 , the peak concentration exceeds the equilibrium approximately by a factor of two [12]. Consequently, under the assumption of equilibrium and lean mixtures, the higher $\mathrm{OH}$ concentrations would be misinterpreted as higher temperatures. However, in instantaneous distributions, flame front regions can be identified relatively easily by their large concentration gradients. If average temperatures are calculated from a larger number of time-resolved measurements, the regions with $\mathrm{OH}$ superequilibrium would contribute to the average signal, resulting in a bias towards higher temperatures. However, because the excess over equilibrium concentrations in pressurized combustion is small and the flame front regions are relatively thin, this effect is small as long as flame front regions are stochastically distributed by turbulence. The requirement of fuel-lean mixtures is of higher importance.

The effect of the equivalence ratio on the temperature-concentration relationship is illustrated in Fig. 1. It shows $\mathrm{OH}$ equilibrium concentrations on a logarithmic scale as a function of temperature and equivalence ratio for methane/air combustion at 6 bar. The concentrations were calculated using the GASEQ [13] code. This figure illustrates the strong dependence of the $\mathrm{OH}$ concentration on temperature, resulting in a high temperature sensitivity of this method. But at the same time it emphasizes the importance of a restricted equivalence ratio range to $\Phi<0.9$ to obtain an unambiguous function $\mathrm{T}([\mathrm{OH}])$, approximately independent of the equivalence ratio. For example, by following the iso-line of $\mathrm{OH}$ concentration starting at $2100 \mathrm{~K}$ and $\Phi=0.3$, it can be seen that the assumption of a constant equivalence ratio leads to a maximum temperature error of 45 degrees (near $\Phi=0.65$ ) or $2 \%$. In Fig. 1 also the adiabatic flame temperatures of the flames examined in the validation experiment (see below) at $\Phi=0.75,0.9$ and 1.1, respectively, of the coflame burner are indicated by arrows. While the flame at $\Phi=0.9$ is just at the upper bound of validity range, the flame at $\Phi=1.1$ as a fuel rich flame is outside of this range. But with an $\mathrm{OH}$ concentration comparable to that of the lean flame at $\Phi=0.75$ it is a good candidate to test the $\Phi$-dependence of the absorptionto-fluorescence ratio or fluorescence quantum yield, discussed below. In addition to the quantities important for the validation experiment, also the operating conditions of an application experiment in an LPP (lean premixed prevaporized) burner [14] for aero 


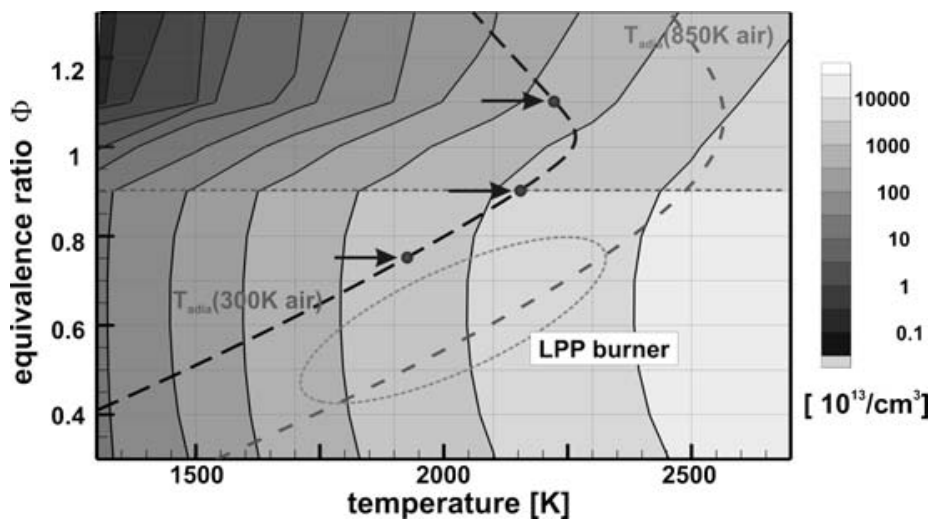

Fig. 1. OH equilibrium concentration as function of temperature $T$ and equivalence ratio $\Phi$, calculated for a methane/air flame at 6 bar. Additionally the adiabetic flame temperatures of flames with non-preheated air $\left(300 \mathrm{~K}\right.$, dark curve) and of flames at take-off conditions with inlet air temperature $T_{3}=850 \mathrm{~K}$ (light curve) are shown. The arrows indicate the adiabatic flame temperatures for the examined flames at $\Phi=$ $0.75,0.9$ and 1.1 of the coflame burner.

engines with air preheating temperatures of about $850 \mathrm{~K}$ are shown (inside of dashed ellipse).

Given an unambiguous temperature - concentration relationship, the task of measuring local temperatures is converted to measuring a local $\mathrm{OH}$ concentration. The following section will give an outline of the theoretical background and describe the experimental procedure. For simplicity, the method is described for a one-dimensional situation. However, it can be easily generalized to a 2D-measurement, since the following discussion holds for each row of pixels in a PLIF image.

The flow chart in Fig. 2 gives an overview of the procedure and the individual steps involved, which are explained in more detail in the following.

As a laser light sheet with a wavelength corresponding to an $\mathrm{OH}$ absorption line passes through a flame, it will create a local LIF intensity $I_{\mathrm{LIF}}(x)$, which can be expressed as [15]

$$
I_{\mathrm{LIF}}(x)=I_{\mathrm{laser}}(x, v) \cdot[\mathrm{OH}(x, T)] \cdot f_{\mathrm{B}, i}(T) \cdot D_{i} \cdot B_{i j} \cdot \eta_{j} \cdot g(x) \cdot \xi
$$

Here, $I_{\text {laser }}(x, v)$ is the local laser intensity, which will in general be a function of wavelength $v$ because the $\mathrm{OH}$ radicals will absorb light only within a part of the spectral laser profile, as long as the laser bandwidth is larger than the molecular linewidth. $f_{\mathrm{B}, i}(T)$ is the Boltzmann factor, or the temperature-dependent fraction of radicals occupying the initial energy level $i$ out of which the laser excitation occurs. $D_{i}$ and $B_{i j}$ are the degeneracy of the initial state and the Einstein $B$ coefficient of the transition $i \rightarrow j$, respectively. $\eta_{j}$ is the fluorescence quantum yield, or the fraction of radicals relaxing by emission of fluorescence radiation, rather than radiationless collisions. The degree of spectral overlap is described by the function $g(x) . \xi$ is a constant containing the cumulative experimental factors contributing to the detection efficiency of the optical system. 


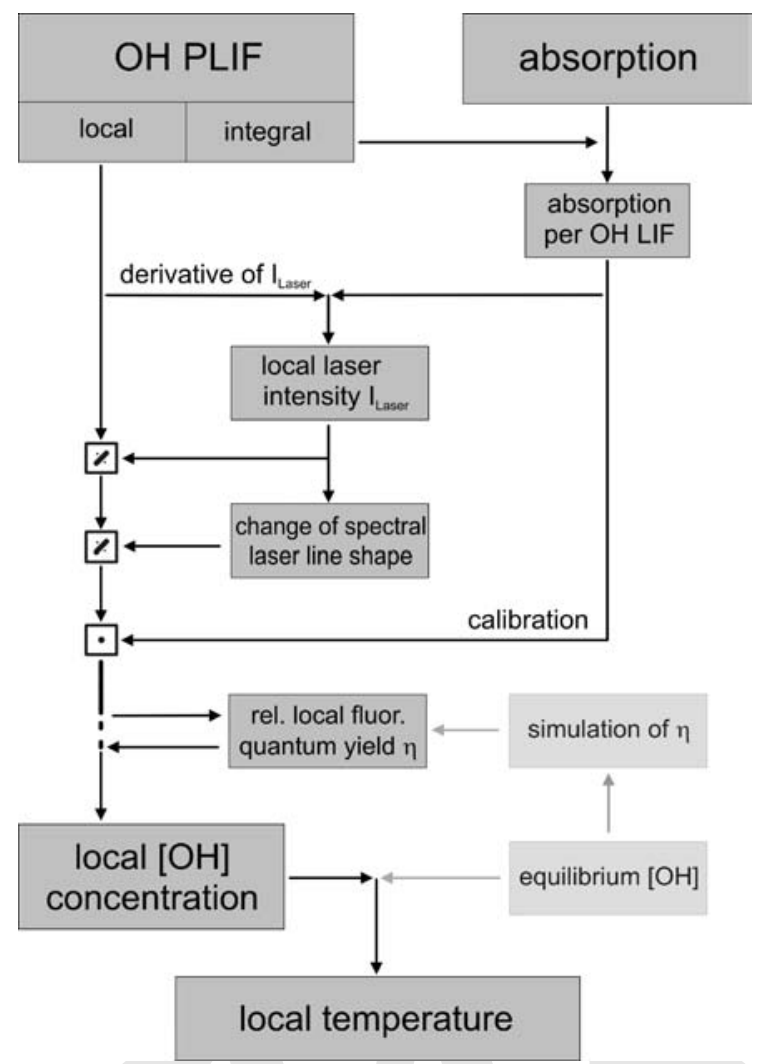

Fig. 2. Flow chart of derivation of temperature information from a combined PLIF/absorption measurement.

During its passage through the flame, the laser intensity will be attenuated according to the Lambert-Beer law:

$$
-d I_{\mathrm{laser}}(x)=\alpha_{\mathrm{laser}}(x) \cdot I_{\mathrm{laser}}(x) d x
$$

With $\alpha_{\text {laser }}(x)$ : absorption coefficient. It will therefore be necessary to reconstruct the local laser intensity from the incident intensity and the absorbed fraction along the path from the entrance of the flame up to position $x$. The laser absorption along a path increment $d x$ is proportional to the local absorption coefficient, which can not be measured directly. However, it can be approximated by the measured local fluorescence intensity $I_{\mathrm{LIF}}(x)$, since each absorbed photon will cause a LIF signal with the probability of the fluorescence quantum yield $\eta(x)$ :

$$
\begin{aligned}
& -d I_{\mathrm{laser}}(x) \cong a_{0} \cdot I_{\mathrm{LF}}(x) d x \\
& a_{0}=\frac{A}{\int I_{\mathrm{LFF}}(x) d x} \cdot I_{\text {laser }}(0)
\end{aligned}
$$


Here, the proportionality constant $a_{0}$ is ratio of the integral absorption $A$ through the entire flame, and the corresponding integrated LIF intensity $\int I_{\mathrm{LIF}}(x) d x$ along the laser beam path through the flame. Thus, the OH LIF image is proportional to the derivative of the laser intensity. This implies another vital condition for the applicability of the combined absorption/PLIF method: The full width of the flame has to be imaged; otherwise, an error will occur due to missing contributions to the integral LIF signal in Eq. (3). All quantities of the right side of Eq. (3) are measured, so that the local laser intensity $I_{\text {laser }}(x)$ can be determined by integration of Eq. (3). The approximation that an integral proportionality constant $a_{0}$ is used instead of a local value $a(x)$, will be discussed later.

The $\mathrm{OH}$ concentration according to Eq. (1) is determined in two steps. First the LIF signal, $I_{\mathrm{LIF}}(x)$ is normalized with respect to the local laser intensity $I_{\text {laser }}(x)$ and to the local spectral overlap $g(x)$ relative to the overlap of the initial incoming laser $g_{0} \equiv g(x=0)$. This change of spectral overlap $g_{\text {rel }}(x)=g(x) / g_{0}$ is caused by a change of the spectral laser line shape due to absorption. If the spectral bandwidth of the exciting laser is in the same order or larger than the width of the molecular transition, then the laser frequencies at the centre of the transition are more strongly absorbed than those at the wings of the transition. The reduction or change of the spectral overlap is simulated as function of the absorption or as function of the remaining local laser intensity $I_{\text {laser }}(x)$, i.e. $g_{\text {rel }}(x)=g_{\text {rel }}\left(I_{\text {laser }}(x)\right)[15]$ :

$$
I_{\mathrm{LF}}^{*}(x)=\frac{I_{\mathrm{LIF}}(x)}{I_{\text {laser }}(x) \cdot g_{\text {rel }}(x)}
$$

In the second evaluation step the calibration of the LIF signal $I_{\mathrm{LIF}}^{*}(x)$ makes use of the relation between absorption and number density [16]:

$$
\begin{aligned}
\left.\mathrm{OH}_{i}(x)\right] \cdot \frac{h v_{j i}}{c} \cdot B_{j i} & =\int \alpha_{j i}(x, v) d v \\
& =\iint \alpha_{j i}\left(x, v-v^{\prime}\right) \cdot h_{\text {laser }}\left(x, v^{\prime}\right) d v^{\prime} d v \\
& =\int \alpha_{j i}^{\text {laser }}(x, v) d v
\end{aligned}
$$

Here $\alpha_{j i}(x, v)$ is the molecular absorption coefficient of the transition $j \leftarrow i$ at the frequency $v . \alpha_{j i}^{\text {laser }}(x, v)$ is defined as the absorption coefficient of the transition convoluted with the spectral line shape of the laser, $h_{\text {laser }}(x, v)$. In the experiment we want to determine the local absorption $\alpha_{\text {laser }}\left(x, v_{0}\right)$ of our laser at the centre frequency $v_{0}$, so by factoring out we can rewrite Eq. (5) in terms of laser absorption and the spectral overlap $g\left(x, v_{0}\right)$ :

$$
\begin{aligned}
{\left[\mathrm{OH}_{i}(x)\right] \cdot \frac{h v_{j i}}{c} \cdot B_{j i} } & =\alpha_{j i}^{\text {laser }}\left(x, v_{0}\right) \cdot \int \frac{\alpha_{j i}^{\text {laser }}(x, v)}{\alpha_{j i}^{\text {laser }}\left(x, v_{0}\right)} d v \\
& =\alpha_{j i}^{\text {laser }}\left(x, v_{0}\right) \cdot g^{-1}\left(x, v_{0}\right) \\
& \cong I_{\mathrm{LIF}}^{*} \cdot a_{0} / g_{0}
\end{aligned}
$$


Table 1. Dependence of fluorescence quantum yield $\eta_{\text {rel }}$ on temperature and equivalence ratio for kerosene/ air combustion at $850 \mathrm{~K}$ preheat temperature and 6 bar.

\begin{tabular}{ccccc}
\hline$\Phi$ & 0.5 & 0.7 & 0.9 & 1.1 \\
$T[\mathrm{~K}]$ & 1971 & 2310 & 2547 & 2631 \\
$\eta_{\text {rel }}$ & 1.14 & $\equiv 1$ & 0.93 & 0.86 \\
\hline
\end{tabular}

According to Eqs. (3) and (4), the local absorption is approximately expressed as a function of the measured local LIF intensity $I_{\mathrm{LIF}}^{*}$. The result shows that the multiplication of the LIF signal $I_{\mathrm{LIF}}^{*}$ by $a_{0}$, the latter being composed of measurable quantities, leads to the $\mathrm{OH}$ number density of the initial quantum state $i$. By this calibration step, the unknown experimental factor $\xi$ and the fluorescence quantum yield $\eta_{j}$ in Eq. (1) are determined. The total number density of $\mathrm{OH}$ is given by dividing Eq. (6) by the Boltzmann factor $f_{\mathrm{B}, i}(T)$ of this state:

$$
[\mathrm{OH}(x)] \cong I_{\mathrm{LIF}}^{*} \cdot \frac{a_{0}}{g_{0} \cdot \frac{h v_{j i}}{c} \cdot B_{j i} \cdot D_{i} \cdot f_{\mathrm{B}, i}(T)}
$$

The Boltzmann factor $f_{\mathrm{B}, i}(T)$ in Eq. (7) is temperature-dependent and therefore $a$ priori unknown; however, by a suitable choice of the initial level for the LIF excitation, this dependence can be kept so small compared to the high temperature dependence of the $\mathrm{OH}$ number density itself that it can be safely ignored. For example, the spectrally overlapping line pair $\mathrm{Q}_{1}(9.5) / \mathrm{Q}_{2}(7.5)$ of $\mathrm{OH}$ [17] has a combined population fraction that varies by less than $10 \%$ in the temperature range $1400-2500 \mathrm{~K}$.

Instead of the local absorption coefficient $\alpha_{\text {laser }}(x)$ in Eq. (2), only the LIF intensity normalized to the mean value $a_{0}$ as ratio of integral absorption and LIF intensity, is used approximately in Eq. (3) and in the further equations. Therefore the total number of $\mathrm{OH}$ molecules along the laser beam path is determined correctly. But the local ratio of fluorescence and absorption, i.e. the fluorescence quantum yield $\eta$ of the $\mathrm{OH}$ transition, can vary around the mean value, which means that there is an uncertainty in the ratio of the local LIF intensity and the corresponding $\mathrm{OH}$ concentration. In general $\eta$ depends on the gas composition and temperature. To estimate the variation, the quantum yield of the $\mathrm{OHAX}(1,0) \mathrm{Q}_{1}(9.5)$ transition mentioned before was calculated using the LASKIN program [18] for typical operating conditions of a kerosene/air flame at 6 bar and $850 \mathrm{~K}$ air preheating, typical for a combustor test rig. The results are given in Table 1.

A local variation of the fluorescence quantum yield is treated iteratively. Assuming conditions of a lean pressurized flame, the unique relationship of $\mathrm{OH}$ equilibrium concentration and gas composition is used to expand the fluorescence quantum yield according to Table 1 with respect to the value of the mean $\mathrm{OH}$ concentration. The preliminary $\mathrm{OH}$ concentration is corrected by the inverse relative quantum yield. The boundary condition of the integral number of $\mathrm{OH}$ radicals measured by the integral absorption is normally fulfilled after the first iteration step within a few percent.

In the final evaluation step the local $\mathrm{OH}$ concentration $[\mathrm{OH}(x)]$ is converted to temperature $T(x)$ using the temperature-concentration relationship calculated for equilibrium conditions [13].

Regarding superequilibirium $\mathrm{OH}$ concentrations, the relaxation of $\mathrm{OH}$ was found to be fast enough in order reach equilibrium conditions within fractions of millimetres. 
For example, for stoichiometric laminar premixed $\mathrm{CH}_{4}$ /air flames, superequilibrium $\mathrm{OH}$ concentrations relax within $0.6 \mathrm{~mm}$ at 5 bar and $0.3 \mathrm{~mm}$ at $10 \mathrm{bar}$, respectively. At realistic gas turbine operation conditions, i.e., at elevated air inlet temperatures and pressures, $\mathrm{OH}$ superequilibrium concentrations indicating the flame front, was observed only in fuel-rich, sooting flames. In lean flames of the LPP burners we never found a clear $\mathrm{OH}$ superequilibrium distribution. So the assumption of equilibrium conditions is suitable for most parts of the flame except the small reaction zone.

\section{Accuracy considerations}

The accuracy consideration of the combined OH PLIF/absorption technique will be subdivided into two parts. The first part addresses the methodical and the experimental uncertainties of the $\mathrm{OH}$ concentration determination. In a second part the accuracy of the subsequent conversion of $\mathrm{OH}$ concentration into temperature under the test conditions of lean premixed pressurized flames will be discussed.

When deriving the $\mathrm{OH}$ concentration from combined laser absorption and PLIF measurements according to Eq. (7), the accuracy is determined by the uncertainties of spectroscopic constants, like the Einstein B coefficient, or the Boltzmann factor $f_{\mathrm{B}, j}$ in the temperature range of interest, and by the uncertainties of the directly measured quantities, like the LIF intensity $I_{\mathrm{LIF}}(x)$ and the integral absorption $A$ of each row of the LIF image, and indirectly measured quantities, like the spectral overlap $g_{0}$ of the laser and the molecular transition at the entrance of the combustor and its change $g_{\text {rel }}$ during passage. The smallest uncertainty of all those quantities has the Einstein B coefficient $( \pm 5 \%)$ as a well known spectroscopic constant $[19,20]$. Choosing a proper transition like the spectrally overlapping line pair $\mathrm{Q}_{1}(9.5) / \mathrm{Q}_{2}(7.5)$, not only the accuracy of the Boltzmann factor $( \pm 8 \%)$ can be optimized, but also the spectrally broader structure of this line pair compared to an isolated line reduces uncertainties of the spectral overlap with the laser line $g_{0}( \pm 10 \%)$ and $g_{\text {rel }}(0-15 \%)$. The high absorption coefficient of the structure leads to high integral absorption values of $40-70 \%$ at typical test conditions. Thus, a low error of the absorption measurement (5-10\%) can be reached, and also the spectral interference of the strong OH LIF signal and the residual kerosene LIF can be minimized. The OH LIF on a high signal level can be measured quite exactly $( \pm 5 \%)$, but the error of weak OH LIF interfering with a strong kerosene LIF will increase drastically. As illustrated in Fig. 4, the contribution of kerosene fluorescence is reduced by the spectral filter centered on the $\mathrm{OH}$ emission. The remaining interference depends, of course, on flame conditions. In the test case shown in Fig. 13, for example, the kerosene fluorescence intensity amounted to $10 \%$ of the OH LIF signal. In summary, the uncertainty of the $\mathrm{OH}$ concentration determination adds up to less than $\pm 20 \%$ of a mean $\mathrm{OH}$ concentration $[\mathrm{OH}(x)]$.

The spatially resolved concentration determination via PLIF instead of a spatially resolved absorption measurement presupposes implicitly the knowledge of the fluorescence quantum yield $\eta(x)$. The "mean" fluorescence quantum yield, defined as the value of the quantum yield weighted by absorption, is determined correctly. So, according to the change of the calculated fluorescence quantum yield with temperature (see Table 1), the determination only of $\mathrm{OH}$ concentrations at very high or very low temperatures 


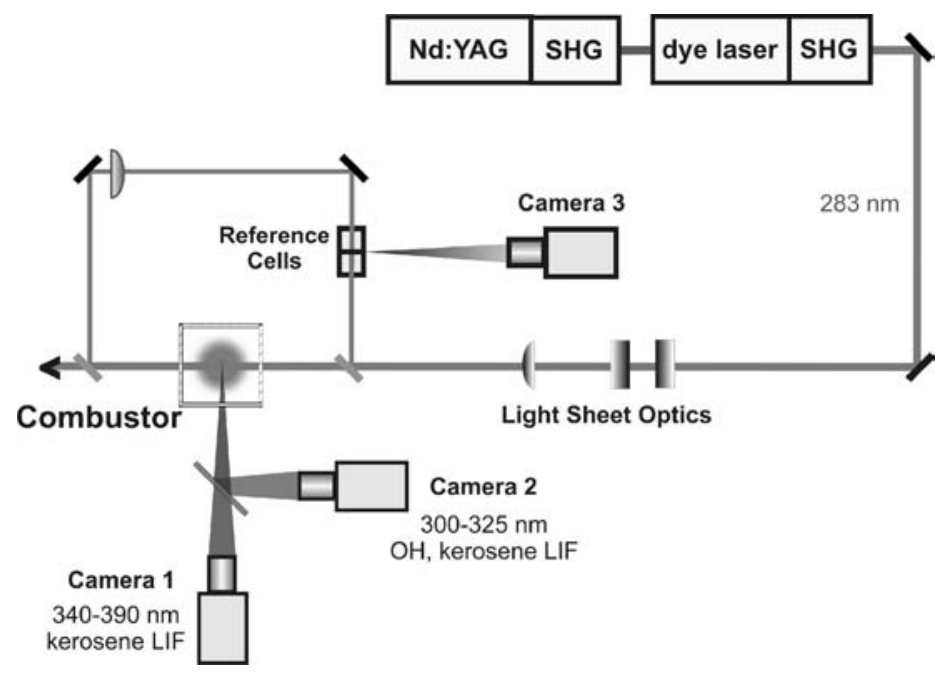

Fig. 3. Setup for combined PLIF and absorption measurements.

could contribute to an additional error. Especially in very lean and consequently cooler regions, the calculated fluorescence quantum yield increases substantially, and an iterative computation of a local quantum yield has to be invoked to reduce a systematic error.

The conversion of $\mathrm{OH}$ concentration to temperature implies an unambiguous relationship of $\mathrm{OH}$ concentration and temperature. This condition is fulfilled in locally lean ( $\Phi \leq 0.9$ ) flames under pressure ( $p \geq 5$ bar), with their fast relaxation to chemical equilibrium conditions even near $(\leq 1 \mathrm{~mm})$ the flame front. An uncertainty of $20 \%$ of the $\mathrm{OH}$ concentration leads to a temperature uncertainty of $40 \mathrm{~K}$ at $2000 \mathrm{~K}$, as a sum of uncertainty of the $\mathrm{OH}$ concentration determination itself and the uncertainty of the conversion to temperature for unknown gas composition for $\Phi \leq 0.9$. A similar estimation leads to an uncertainty of $60 \mathrm{~K}$ for $2300 \mathrm{~K}$ and $40 \mathrm{~K}$ at $1500 \mathrm{~K}$.

\section{Experimental procedure}

The setup used for a combined PLIF/absorption measurement is shown schematically in Fig. 3. The figure shows a "top view", i. e., the laser light sheet extends in the direction perpendicular to the drawing plane. Laser radiation at wavelengths around $283 \mathrm{~nm}$, corresponding to the spectral range of the $\mathrm{OH} X^{2} \Pi \rightarrow A^{2} \Sigma\left(v^{\prime \prime}=0 \rightarrow v^{\prime}=1\right)$ transition [17], is generated by a combination of a pulsed, frequency-doubled Nd:YAG laser and a frequency-doubled dye laser [21]. This laser technology is well-established and has been shown to work reliably even under adverse environmental conditions frequently prevailing near large test rigs [22]. The UV beam is shaped into a light sheet of typically $50 \mathrm{~mm}$ height and a waist thickness around $200 \mu \mathrm{m}$, which is usually the limiting dimension for the spatial resolution of the method. The pixel resolution of the cameras was typically between $200 \mu \mathrm{m}$ and $400 \mu \mathrm{m}$, depending on the experiment. 


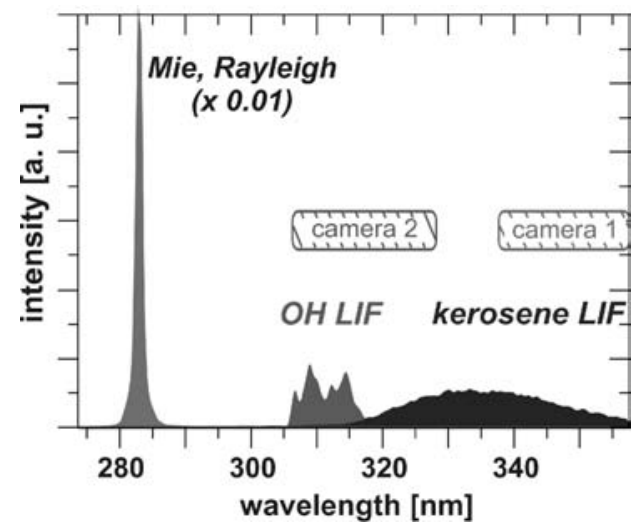

Fig. 4. Emission spectrum in a kerosene flame following excitation of $\mathrm{OH}$ near $283 \mathrm{~nm}$. The hatched regions mark the detected range for camera 1 (pure kerosene) and camera 2 ( $\mathrm{OH}$ and kerosene).

Since these experiments usually serve to investigate global aspects of the flame structure and are not intended to address turbulence-chemistry interactions on small spatial scales, this resolution is by far sufficient for this purpose.

Before entering the combustion chamber, a small portion of the UV light (usually about $1 \%$ ) is split off the main beam and directed into a quartz cell filled with highly diluted laser dye, causing it to fluoresce. The cell is denoted as "reference cells" in Fig. 3. The intensity of the fluorescence, which is recorded by camera 3, is proportional to the incident laser radiation; therefore, the fluorescence intensity distribution can be used to normalize the PLIF intensity from the flame with respect to the local laser intensity.

The light sheet then passes through the flame, and the same fraction of transmitted light as on the entrance side of the burner is guided to a second reference cell identical to the one measuring the intensity distribution of the incident light. The two cells are placed side by side, so that camera 3 records the intensity distribution of the incident and the transmitted light simultaneously. Therefore, the two reference cells serve a twofold purpose: They measure the - usually non-uniform - intensity distribution across the incident light sheet, and they are used to measure the spatially integrated absorption by the flame, which is needed to calculate the number of absorbing molecules along the direction of propagation of the laser light through the flame for each pixel row, as outlined in Sect. 2.

The resulting laser-induced fluorescence is monitored at a right angle to the laser beam propagation by the two image intensified UV-sensitive ICCD cameras 1 and 2 . A dielectric beam splitter is located in front of the two cameras, which performs a spectral separation of the detected light. The reflected part of the fluorescence, recorded by camera 2, covers the wavelength range between 300 and $325 \mathrm{~nm}$. This range contains the entire $\mathrm{OH}$ fluorescence, as well as part of the LIF resulting from aromatic compounds contained in kerosene. This means that the $\mathrm{OH}$ fluorescence detected by camera 2 is in general contaminated by unwanted kerosene LIF. Camera 1 records the spectral range above $340 \mathrm{~nm}$, which results from LIF of kerosene only, with no contribution of $\mathrm{OH}$. The spectra shown in Fig. 4 illustrate the wavelength regions where signals result- 
ing from elastic scattering (Mie, Rayleigh), as well as $\mathrm{OH}$ and kerosene fluorescence occur. If the laser wavelength is tuned off an absorption line of $\mathrm{OH}$, only the continuous broadband absorption of kerosene leads to the emission of a LIF signal, which is detected by both cameras at usually different sensitivities, because the fluorescence intensities transmitted (camera 1) or reflected (camera 2) by the beam splitter for the respective wavelengths, as well as the camera sensitivities are not identical. These "off resonance" signals can be used to normalize the kerosene signal intensity recorded by camera 2 with respect to camera 1 . It allows calculating the fraction of detected intensity in the image of camera 2 which has to be subtracted because it results from kerosene rather than $\mathrm{OH}$ LIF.

The subtraction of a kerosene contribution in the total PLIF signal in addition to the $\mathrm{OH}$ fluorescence is in general an additional source of statistical error. However, it should be kept in mind that by definition, the applicability of the temperature measurement technique is limited to scenarios where the imaged area is fuel-lean; therefore, the kerosene contribution to the LIF signal can be expected to be small.

This subtraction procedure of the kerosene LIF background is justified only if the image areas of both cameras are identical on a pixel-by-pixel basis. This requires careful mechanical alignment of both cameras, as well as possibly a software superposition of the image areas by warping procedures, because pincushion or other image deformations caused by camera lenses, image intensifiers or transmitting optics are usually different for individual cameras and can not be compensated by mechanical adjustments alone. The unavoidable residual mismatch after mechanical superposition of the camera images was removed by recording a calibration target pattern with each camera and using a warping algorithm provided by the camera manufacturer. The warping parameters were derived from approx. 120 reference points evenly distributed over the image area.

The OH LIF images, which are obtained after subtraction of kerosene LIF offset and normalization with respect to the sheet intensity distribution recorded by camera 3 , are then further processed according to the procedures described in Sect. 2.

\section{Validation experiments}

Before the method outlined above is applied under conditions typical for gas turbine combustors, it is necessary to validate it under simplified conditions. Gas turbine combustion is characterized by highly turbulent swirling flows with large temporal and spatial gradients of temperature, hence also $\mathrm{OH}$ concentration, as well as flow velocities and gas composition; an assessment of the applicability and accuracy of the thermometry technique should be performed in a better controlled environment. The OH PLIF image in the middle part of Fig. 13, which was recorded in a single sector combustor with realistic nozzle geometry and flow field, gives an impression of the structures prevailing in a real gas turbine flame.

A key conclusion of the discussion above was that two requirements have to be fulfilled to allow application of this method: Lean mixtures and chemical equilibrium at every location in the observation plane. The aim of this chapter is to prove the applicability of the technique where such conditions prevail, and the assessment of the 
effect of deviations from the two conditions on the reliability of planar temperature measurements.

Therefore, the OH-PLIF-based temperature validation measurements were performed in a premixed laminar flame at elevated pressure. Such a flame provides steady conditions necessary for reference measurements by other techniques which can not be performed in real time; it can be adjusted to fulfil the requirements regarding equilibrium and equivalence ratio mentioned before, and at the same time represents conditions typically for lean gas turbine combustion in terms of pressure and equivalence ratio range. The "coflame" burner of the Institute of Combustion Technology of the DLR in Stuttgart [23,24] allows independent control of pressure and equivalence ratio.

Because of its unequalled accuracy, the $\mathrm{N}_{2}$ CARS measurement technique [1] was chosen as an independent technique to determine reference temperatures. The high quality CARS temperature measurements are performed at selected positions in the premixed laminar flame of the high pressure "coflame" burner. These temperatures are used to validate the $\mathrm{OH}$ concentration and temperature measurements. The results of the PLIF measurement technique is compared with the results of the CARS reference measurements for different flame conditions. Starting from a slightly lean standard flame ( $\Phi=0.9$ ) at 6 bar, the results of a pressure variation (2-8 bar) and of a variation of the equivalence ratio inside and outside the lean regime $(\Phi=0.75-1.1)$ are presented and discussed.

\section{The "coflame" burner}

To validate the OH PLIF temperature measurements the high pressure "coflame" burner of the Institute of Combustion Technology of the DLR in Stuttgart was chosen, because it meets the requirements of the validation experiment in a very good approximation. A methane/air flame is stabilized on a sintered matrix with $41 \mathrm{~mm}$ diameter. It is surrounded by a shielding flame which reduces heat losses and further oxidation of the inner flame. This shielding or coaxial flame gave the burner its name. In a wide pressure range the burner produces stable premixed laminar flames, which are very homogeneous in radial direction [23,24]. Figure 5 shows the $\mathrm{CH}_{4}$ /air premixed laminar flame at 6 bar; the structure of the "inner flame" and the surrounding "shielding flame" can be seen clearly. The inner flame to be studied was stabilized above a water-cooled sintered bronze matrix (see Fig. 6). This flame was surrounded by the "shielding flame", also stabilized on a bronze ring matrix. The purpose of the outer flame was to shield the inner flame from cold surroundings and oxidation. Additionally, the shielding flame could help to stabilize the inner flame, like a pilot flame. These two flames were surrounded by an air coflow. The diameters of the inner matrix, the ring matrix and the coflow duct were $41.3 \mathrm{~mm}, 61.3 \mathrm{~mm}$, and $150 \mathrm{~mm}$, respectively.

Flame stability, specifically at increased pressure, is difficult to achieve. That is mainly due to different cold and hot gas velocities of the three fluids inner mixture, ring mixture and coflowing air. While the coflow air remains cold, both combustion mixtures accelerate in the flame front soon after the burner surface. Best, although not perfect, flame stability balancing the outer, non-accelerated air flow with the coannular fluid mixtures was achieved with different cold gas velocities for the combustible mixtures, 


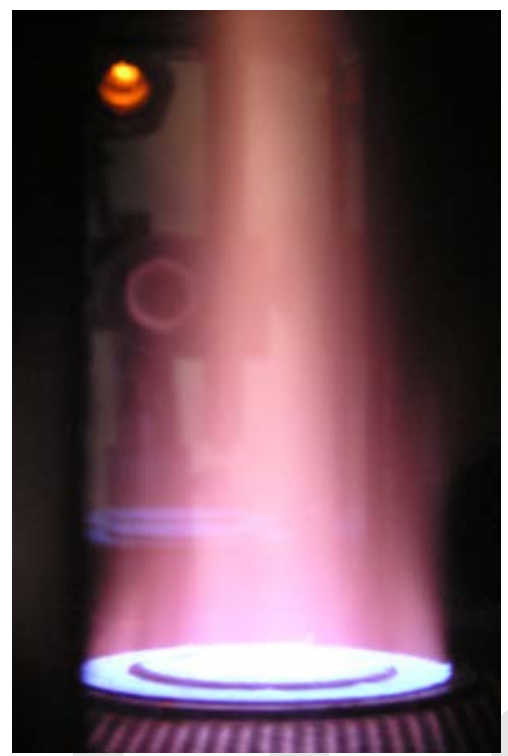

Fig. 5. Premixed laminar flame for comparison of OH PLIF temperature images with CARS measurements.

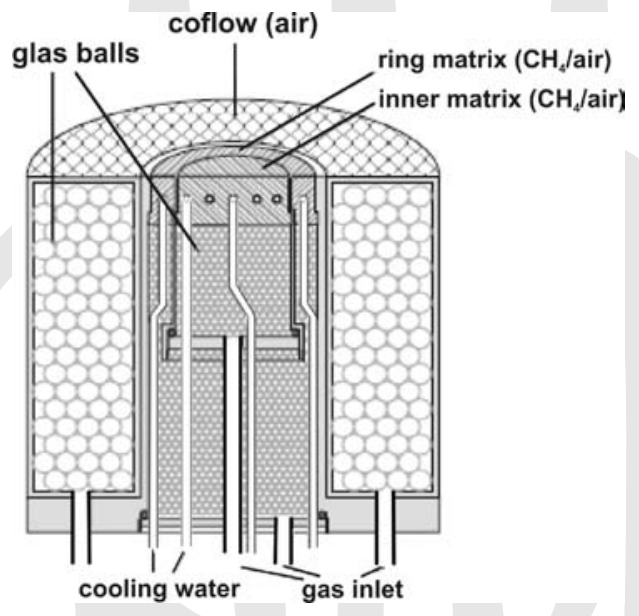

Fig. 6. Mechanical setup of the coflame burner.

while different equivalence ratios were not considered compatible with the target of the study. Given the equal stoichometry of both flames and the flame fronts being close to the burner surface, diffusion between the flames is considered negligible. This approach resulted in flames that were very stable in the monitored regions.

All gas flows were controlled using electronic mass flow controllers (Bronkhorst $\mathrm{Hi}-\mathrm{Tec}$ ), which were calibrated in-house using Coriolis-effect mass flow meters (Danfoss). The reproducibility was better than $0.5 \%$ of the maximum flow rate. 
Table 2. Flame settings for the investigated flames.

\begin{tabular}{|c|c|c|c|c|c|c|c|c|c|c|}
\hline \multirow[b]{2}{*}{$\begin{array}{l}\text { Flame } \\
\quad \#\end{array}$} & \multirow[b]{2}{*}{$\begin{array}{c}\text { Pressure } \\
p \text { [bar] }\end{array}$} & \multicolumn{4}{|c|}{ Inner flame } & \multicolumn{4}{|c|}{ Shielding flame } & \multirow{2}{*}{$\begin{array}{c}\text { Coflow } \\
\text { Air } \\
\text { [sl/min] }\end{array}$} \\
\hline & & $\Phi_{\text {inner }}$ & $\begin{array}{c}\mathrm{CH}_{4} \\
{[\mathrm{sl} / \mathrm{min}]}\end{array}$ & $\begin{array}{c}\text { Air } \\
{[\mathrm{sl} / \mathrm{min}]}\end{array}$ & $\begin{array}{c}v_{\text {inner }} \\
{[\mathrm{cm} / \mathrm{s}]}\end{array}$ & $\Phi_{\text {shield }}$ & $\begin{array}{c}\mathrm{CH}_{4} \\
{[\mathrm{sl} / \mathrm{min}]}\end{array}$ & $\begin{array}{c}\text { Air } \\
{[\mathrm{sl} / \mathrm{min}]}\end{array}$ & $\begin{array}{c}v_{\text {shield }} \\
{[\mathrm{cm} / \mathrm{s}]}\end{array}$ & \\
\hline 1 & 6 & 0.9 & 7.79 & 82.62 & 20 & 0.9 & 6.08 & 64.49 & 15 & 564 \\
\hline 2 & 6 & 1.1 & 12.14 & 105.38 & 26 & 1.1 & 7.29 & 63.28 & 15 & 552 \\
\hline 3 & 6 & 0.75 & 5.27 & 67.06 & 16 & 0.75 & 5.14 & 65.43 & 15 & 528 \\
\hline 4 & 1 & 0.9 & 1.82 & 19.28 & 28 & 0.9 & 1.69 & 17.91 & 25 & 120 \\
\hline 5 & 2 & 0.9 & 3.12 & 33.05 & 24 & 0.9 & 2.7 & 28.66 & 20 & 186 \\
\hline 6 & 4 & 0.9 & 5.71 & 60.59 & 22 & 0.9 & 4.32 & 45.86 & 16 & 372 \\
\hline 7 & 8 & 0.9 & 10.38 & 110.16 & 20 & 0.9 & 7.57 & 80.26 & 14 & 570 \\
\hline
\end{tabular}

The stainless steel pressure casing was water-cooled and allowed optical access from four sides by window sizes of $60 \mathrm{~mm}$ width and $120 \mathrm{~mm}$ height. As illustrated in Fig. 5, the entire flame was visible through the windows, ensuring the required unobstructed optical access mentioned before. The pressure in the combustion chamber was controlled by a variable valve at the combustor exit.

The flames studied in this investigation are summarized in Table 2. Flame 1 was the standard flame, just in the lean regime with $\Phi=0.9$. This equivalence ratio represents the theoretical upper limit of the applicability of the thermometry method, resulting from the requirements discussed in the introduction. For this, but also for all the other flames, $\Phi_{\text {inner }}$ was the same as $\Phi_{\text {shield }}$ to achieve a high degree of radial homogeneity. In flames 2 and 3, the equivalence ratio $\Phi$ was varied to be inside and outside the lean regime, i.e. $\Phi=0.75$ and $\Phi=1.1$, while for the flames $4-6$ the pressure was varied between 1 bar and 8 bar at the fixed standard equivalence ratio of $\Phi=0.9$. The gas flow velocities $v_{\text {inner }}$ and $v_{\text {shield }}$ were optimized to get a stable flame with a flame temperature near the adiabatic value.

\section{CARS reference temperature measurements}

CARS (Coherent Anti-Stokes Raman Scattering) spectroscopy has been established in the past as a laser-based, non-intrusive temperature measuring method for combustion processes in practical environments [21,23]. The CARS signal is coherently generated and emitted in a laserlike beam. Using a broadband CARS technique allows to detect an entire $\mathrm{N}_{2}$ Q-branch spectrum in a "snapshot" generated with a single laser pulse lasting about $10 \mathrm{~ns}$. Because the spectral distribution of the Q-branch reflects the population of the rotational and vibrational states, the temperature can be determined directly from the spectrum without calibration. Usually, the temperature is derived from the comparison of the shape of computed and experimental $\mathrm{N}_{2}$ Q-branch spectra. Single shot measurements can be carried out with the repetition rate of the laser $(10 \mathrm{~Hz})$. Typically, a series of 1200 single shot spectra was recorded at each measuring location. Because the experiments were performed in a laminar premixed flame, each series of single shot spectra was averaged. For each averaged spectrum a contour fit of a theoretical spec- 
trum to experimental data was performed to deduce the temperature. A more detailed description of the data evaluation procedure is found in Ref. [25]. The temperature accuracy of the mobile CARS system was tested in an isothermal furnace for single shot measurements and is in the range of $\pm 2.5-3 \%$.

The CARS apparatus consists of a pulsed Nd:YAG laser operated at $\lambda=532 \mathrm{~nm}$ with a repetition rate of $10 \mathrm{~Hz}$ which pumps a narrowband dye laser at $\lambda=591 \mathrm{~nm}$ and a broadband dye laser at $\lambda=685 \mathrm{~nm}$. The folded BOXCARS arrangement was employed to overlap the exciting laser beams at the measuring location. In the overlapping volume the CARS signal is generated and it thus represents the size of the measuring volume and the spatial resolution. The spatial resolution is defined as the length of the measuring volume in beam direction in which $95 \%$ of the CARS signal is generated. From a measurement without flame the spatial resolution was found to be $\mathrm{L}(95 \%)=4 \mathrm{~mm}$. The diameter of the probe volume was approx. $200 \mu \mathrm{m}$. The signal receiving optics at the opposite side of the combustion chamber separated the CARS signal from the laser beams and focused it into an optical fiber with a core diameter of $600 \mu \mathrm{m}$ and a length of $20 \mathrm{~m}$. At the end of the fiber the signal was coupled into a spectrometer and detected with an intensified gateable CCD camera.

For this investigation, the spatial positions of the CARS measurements were defined at the centerline of the flames at different heights above the burner (HAB). They were in the plane of the OH PLIF measurements, at HAB $=5 \mathrm{~mm}, 10 \mathrm{~mm}, 20 \mathrm{~mm}$ and $40 \mathrm{~mm}$, respectively.

\section{OH PLIF/absorption measurements}

In this validation experiment premixed methane/air flames were used, so camera 1 (in Fig. 3) detecting potential spectral interferences of the kerosene LIF was dispensable. To optimize the energy stability, the laser system had to run at UV pulse energies of at least $5 \mathrm{~mJ}$. Under such conditions, and with the sheet parameters as descripbed before, the LIF signal may be partly saturated even at elevated pressures. However, the conversion of LIF intensities to $\mathrm{OH}$ concentrations requires linear excitation, i.e., the laser intensity has to be sufficiently low for the LIF signal to remain proportional to the exciting light intensity. To avoid saturation effects, the UV pulse energy is reduced by reflecting attenuators $(2 \times, 4 \times, 8 \times)$, by an over-expanding light sheet optics producing a light sheet of twice the required height, of which only the central part is used, and by an increased waist thickness of $300 \mu \mathrm{m}$ FWHM. The latter was achieved by a negative cylindrical lens just in front of the combustion chamber. The UV laser bandwidth, which also affects the degree of saturation, was determined to be $0.3 \mathrm{~cm}^{-1}$ FWHM by fitting simulated $\mathrm{OH}$ excitation spectra with laser bandwidth as one adjustable parameter to measured spectra.

The resulting laser-induced fluorescence is spectrally filtered by an $\mathrm{OH}$ interference filter $(305-325 \mathrm{~nm})$ and monitored at a right angle to the laser beam propagation by the ICCD camera 2 (LaVision Flame Star 2) through a $f=100 \mathrm{~mm}$ UV lens with F/2 (Halle). The spatial resolution of the $\mathrm{OH}$ LIF image is $240 \mu \mathrm{m} \times 240 \mu \mathrm{m}$ per pixel. The $\mathrm{OH}$ LIF images, as well the images of the reference cells, were digitized with 14 bit resolution and stored on single-shot basis. For each PLIF measurement of a particular 


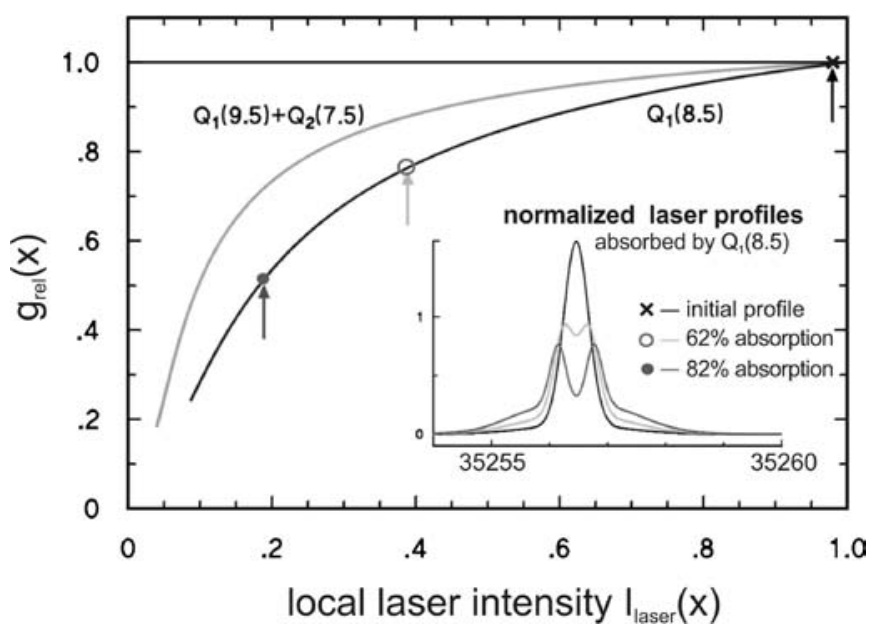

Fig. 7. Relative overlap $g_{\text {rel }}(x)$ of laser and absorption lines as function of local laser intensity calculated for the spectrally isolated line $Q_{1}(8.5)$ and for the double line $Q_{1}(9.5) / Q_{2}(7.5)$.

$\mathrm{OH}$ excitation line, two series of 200 single pulse images were recorded. Between these two "on-resonant" series, an "off-resonant" series with the laser wavelength shifted off an $\mathrm{OH}$ absorption line was taken to get a reference image with no $\mathrm{OH}$ laser absorption (camera 3). The contribution of the $\mathrm{OH}$ chemiluminescence signal (camera 2) to the $\mathrm{OH}$ LIF signal could be neglected.

For the $\mathrm{OH}$ concentration measurements under these experimental conditions, the excitation of the spectrally overlapping line pair $Q_{1}(9.5) / Q_{2}(7.5)[17,26]$ at $35210.45 \mathrm{~cm}^{-1}$ is very attractive for three reasons. First, this line structure is temperature insensitive, i.e. the combined population fraction varies by less than $10 \%$ in the temperature range 1400-2500 K. Secondly, the high absorption coefficient causes laser absorption values of $30 \%$ to $70 \%$. In this absorption range the error of concentration determination due to experimental uncertainties minimizes [27].

Finally the spectral line spacing of $0.5 \mathrm{~cm}^{-1}$ [26] combined with Doppler and pressure broadening [28] leads to a broad, top-head like absorption line structure, for which the experimental uncertainties of the laser line shape affects the spectral overlap $g_{0}=g\left(I_{0}\right)$ of absorption and initial laser line to a small extent only. Consequently, also the spectral overlap $g_{\text {rel }}(x)=g\left(I_{\text {laser }}(x)\right) / g_{0}$, of the absorption and the partially absorbed laser line is less sensitive to the local laser intensity $I_{\text {laser }}(x)$. Figure 7 shows the calculated function $g_{\text {rel }}(x)$ as a function of $I_{\text {laser }}(x)$ for two different laser absorptions, the spectrally isolated absorption line $\mathrm{Q}_{1}(8.5)$ and for the broad double structure $\mathrm{Q}_{1}(9.5) / \mathrm{Q}_{2}(7.5)$. For a laser line, initially with the same width as the isolated line $\mathrm{Q}_{1}(8.5)$, the spectral laser profiles changes with decreasing local laser intensity $I_{\text {laser }}(x)$, so that also the overlap with the absorption line decreases. The decrease of the overlap $g_{\text {rel }}(x)$ is substantially smaller for the absorption by the double structure $\mathrm{Q}_{1}(9.5) / \mathrm{Q}_{2}(7.5)$ than for the absorption by the single line $\mathrm{Q}_{1}(8.5)$.

Equation (7) is used to determine approximately (apart from fluorescence quantum yield effects discussed later) the $\mathrm{OH}$ number density $[\mathrm{OH}(x)]$. To correlate temperature 
and $\mathrm{OH}$ concentration, the combustion equilibrium program GASEQ [13] was used to calculate $\mathrm{OH}$ concentrations, as illustrated in the discussion of Fig. 1.

\section{Results and discussion}

\section{"Coflame" burner tests}

The simultaneous OH LIF and absorption measurements were performed in all flames listed in Table 2. At least two series, each with 200 "on-resonance" single pulse images were taken, between which 200 "off resonance" images were recorded.

The essential steps of the concentration determination are illustrated exemplary in Fig. 8. A typical pair of single pulse images of the OH LIF and reference cells was chosen. It belonged to an "on-resonant" series exciting the $\mathrm{Q}_{1}(9.5) / \mathrm{Q}_{2}(7.5)$ double line at $35210.45 \mathrm{~cm}^{-1}$. The exciting laser passed the flame from left to right, the non-uniform intensity distribution of the laser light sheet is evident. Consequently, in a first step, the LIF image was normalized with respect to the camera sensitivity and to the intensity profile of the incident laser beam, measured by the reference cell in front of the combustor (first row). The resulting $\mathrm{OH}$ image $\mathrm{LIF}_{\text {norm }}$ displays clearly the absorption of the exciting laser. The value of the integral laser absorption A was determined by the ratio of the outgoing and the incoming laser intensities, normalized to the analogue ratio of the averaged "off resonance" image to filter out the absorption caused by $\mathrm{OH}$ only. The "LIF ${ }_{\text {norm }}$ " image is proportional to the derivative of the laser intensity, assuming a uniform fluorescence quantum yield along the path of the laser beam. So with the integral and differential absorption measurements, the local laser intensity could be reconstructed by integration of the LIF signals along the direction of laser beam propagation, according to the procedure described in Eq. (3); it is shown in the third row of Fig. 8. The absorption corrected LIF image LIF $_{\text {abs }}$ in the same row shows a uniform intensity distribution within the entire inner flame. The small remaining horizontal structure is mainly caused by an experimental inadequacy of the beam guidance of the transmitted laser light sheet - one of the bending mirrors was too small for the beam displacements caused by thermal beam steering effects. At this step, the absorption correction of the LIF signal is normally finished. The corrected LIF image seems to provide an image proportional to the $\mathrm{OH}$ distribution. But this image will be changed one more time in the following step.

To calibrate the $\mathrm{LIF}_{\text {abs }}$ image in terms of corresponding $\mathrm{OH}$ number densities, the image has to be multiplied by the ratio $a_{0}$ of integral absorption to the integral LIF signal along the laser path through the flame, according to Eq. (7). This value decreased between $1 \mathrm{~mm}$ and $40 \mathrm{~mm}$ above the flame front by $20 \%$, and consequently also the $\mathrm{OH}$ concentrations decreased nearly linearly with height above the burner. The temperature distribution was deduced using the calculated $[\mathrm{OH}]-T$ correlation (Fig. 1) for the lean flame with $\Phi=0.9$. As shown in the last row of Fig. 8, the temperature distribution in a single-pulse image shows a low noise level, due to the high temperature sensitivity of the $\mathrm{OH}$ concentration. For example, within the small areas of $3 \times 3 \mathrm{~mm}^{2}$ around the dots indicating the points of CARS reference measurements the "pixel noise" of the temperature is less than $14 \mathrm{~K}$. The temperatures deduced from the $\mathrm{OH}$ concentrations decrease from $2110 \mathrm{~K}$ just above the flame front to $2050 \mathrm{~K}$ at $40 \mathrm{~mm}$ height above 

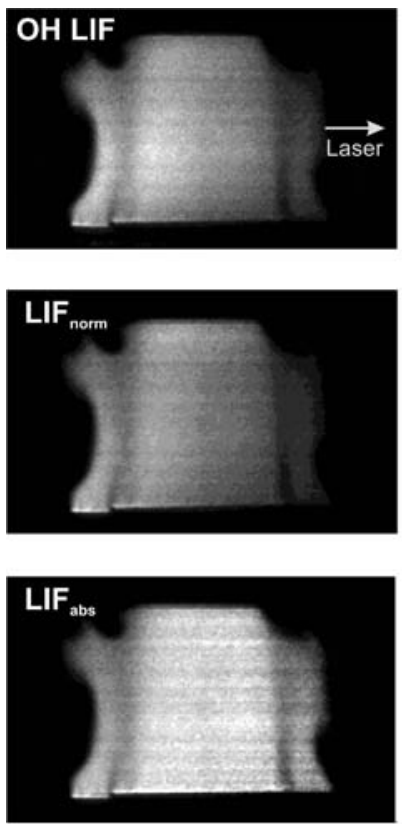

\section{$[\mathrm{OH}]$}
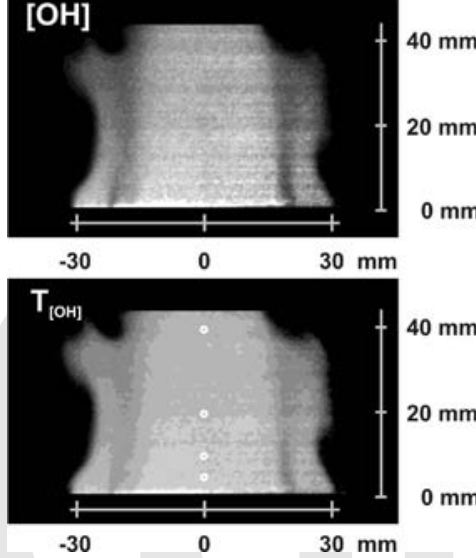

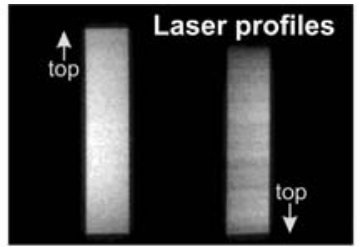

in front and beyond the combustor

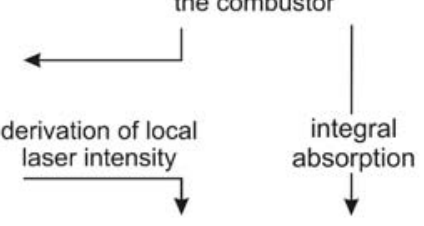

local laser intensity
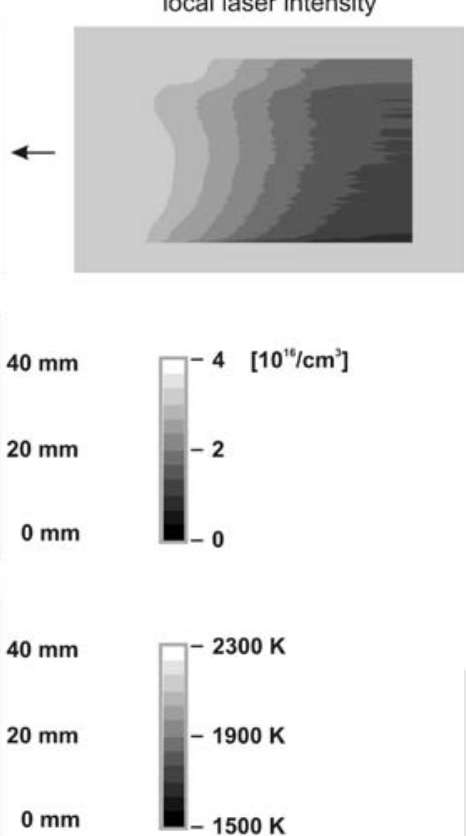

Fig. 8. Example of temperature determination from a single-pulse OH PLIF image (1st row). The integral absorption measurement together with the LIF image (2nd row) were used to reconstruct the local laser intensity (3rd row) and to calibrate the $\mathrm{OH}$ concentration image (4th row), which correlated with temperature for the lean $\mathrm{CH}_{4}$ /air flame 1 at $\Phi=0.9$ and $p=6$ bar (5th row).

the matrix. Just $1 \mathrm{~mm}$ above the burner matrix a high $\mathrm{OH}$ concentration indicates a $\mathrm{OH}$ super-equilibrium in the region of the flame front. The high $\mathrm{OH}$ concentration is interpreted as an about $100 \mathrm{~K}$ higher temperature by the evaluation algorithm, because it is based on the assumption of thermal equilibrium and can not account for nonequilibrium effects. With increasing pressure, relaxation rates to equilibrium increase 


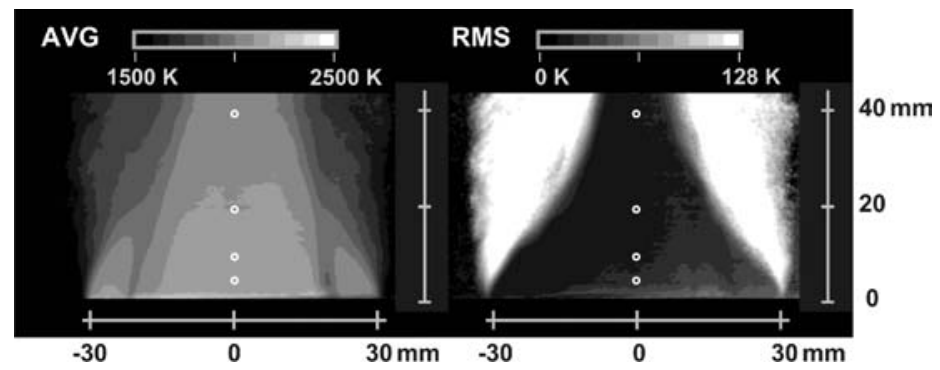

Fig. 9. Temperature distribution of the lean $\mathrm{CH}_{4}$ /air flame 1 ( $\Phi=0.9, p=6$ bar) and the RMS values determined from 200 single-pulse $\mathrm{OH}$ concentration measurements. The dots at the centerline indicate the positions of the reference CARS measurements.

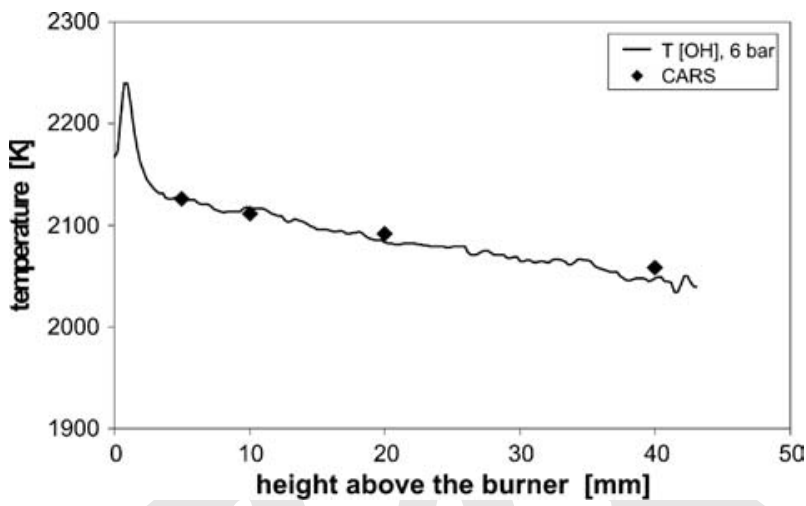

Fig. 10. Axial temperature profiles of the standard flame 1 at 6 bar, equivalence ratios $\Phi=0.9$, determined by $\mathrm{OH}$ concentration measurements and by CARS.

and lead to a reduction of systematic errors caused by thermal nonequilibrium. The pressure effect on equilibrium concentrations and the associated effect on systematic temperature errors will be discussed later in the context of experiments at varying pressures; at this point, the single-pulse capability and the reproducibility of the technique are demonstrated.

The average and the standard deviation of the 200 single-pulse temperature images were calculated, and the results are shown in Fig. 9. It is remarkable that for the stable region of the flame the temperature distribution from a single-pulse image in Fig. 8 agrees very well with that of the averaged image. Or, looking at the RMS image, the standard deviation of the temperatures is very low in the region of high flame stability. At the centre line of the flame the RMS values are $20 \mathrm{~K}$, mainly caused by the inadequate experimental setup - see above - to correct for the beam steering effects.

To prove the reproducibility of the method, temperatures were measured in this flame on consecutive days several times. A total of 11 independent measurements resulted in very low RMS values, always less than $13 \mathrm{~K}$ within the stable area of the flame. 


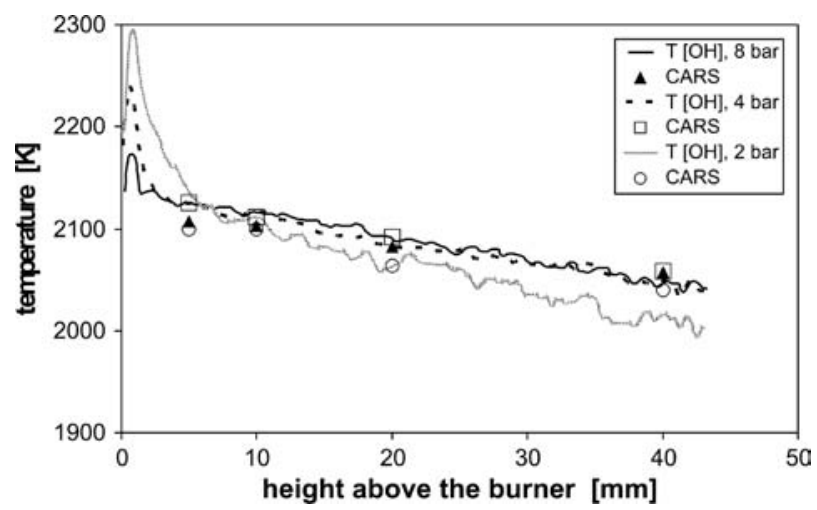

Fig. 11. Axial temperature profiles of flames at $\Phi=0.9$ and pressures of 2 bar (light grey), 4 bar (dotted), and 8 bar (solid), determined by $\mathrm{OH}$ concentration measurements (lines) and by CARS measurements (symbols).

The averaged RMS value of $10 \mathrm{~K}$ illustrates the short and long term stability of the coflame burner and the reproducibility of the concentration measurements.

The dots at the flame centreline in Fig. 9 indicate the positions of the reference CARS temperature measurements. They are at 5, 10, 20, and $40 \mathrm{~mm}$ height above the burner (HAB), respectively. Figure 10 shows the temperature profiles at the flame centreline, determined by the $\mathrm{OH}$ concentration measurement and by the reference CARS measurements. The agreement of the temperature profile deduced from the $\mathrm{OH}$ concentration measurements and the CARS temperatures is excellent. The slow temperature decrease with increasing HAB is well captured by both methods. The apparent temperature peak measured by PLIF near the flame front $1 \mathrm{~mm}$ above the burner is caused by $\mathrm{OH}$ superequilibrium as discussed above, but the concentrations relax to chemical equilibrium within 1-2 $\mathrm{mm}$.

The pressure influence on the temperature distribution using $\mathrm{OH}$ concentration measurements was examined by pressure variation between 2 bar and 8 bar, as shown in Fig. 11. The equivalence ratio of all flames was fixed at $\Phi=0.9$ to obtain an approximately constant flame temperature. As expected, the measured temperature distributions of the flames are similar. The main differences appear just above the burner matrix. The $\mathrm{OH}$ superequilibrium concentration relaxes significantly faster at 8 bar than at 2 bar. For reasons of clarity only profiles for three pressures are represented. The omitted profiles at 6 bar were shown in Fig. 10. Looking only at the CARS temperature profiles of Figs. 10 and 11, they show comparable temperatures with no significant pressure effect. The agreement of $\mathrm{OH}$ temperatures and the reference CARS temperatures is excellent, within the accuracy of $2.5 \%$ for CARS measurements, or the systematic uncertainty of $60 \mathrm{~K}$ from the $\mathrm{OH}$ concentration measurement. The latter uncertainty corresponds to the assumption of a constant equivalence ratio in the lean regime, or, in other words, ignoring the small residual $\Phi$ dependence of the $\mathrm{OH}$ concentration in lean flames at equilibrium. The high $\mathrm{OH}$ superequilibrium concentrations near the burner matrix are interpreted as non-physical high temperatures in the flame front. With increasing pressure, i.e. with increasing relaxation rates, the $\mathrm{OH}$ equilibrium concen- 


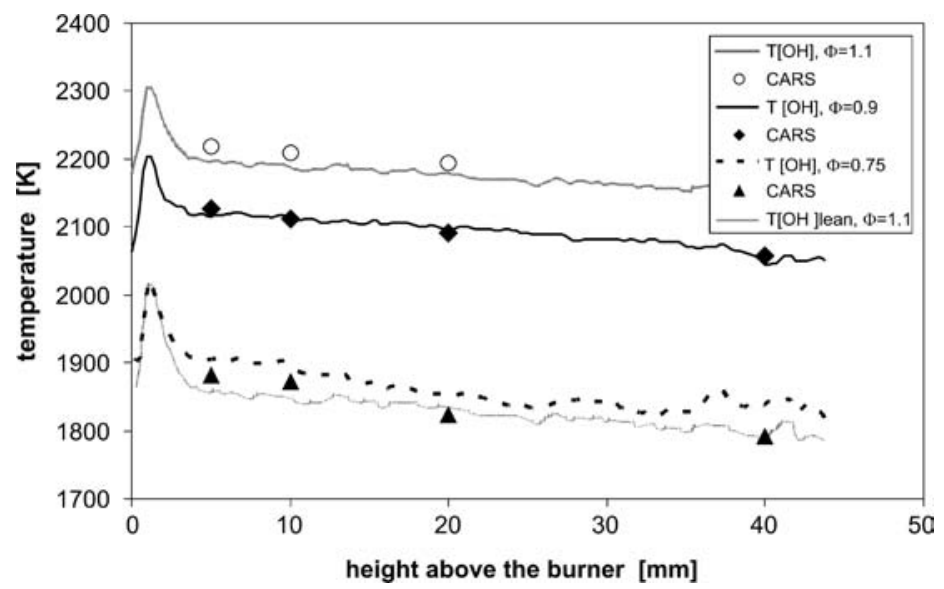

Fig. 12. Axial temperature profiles at three different equivalence ratios and $p=6$ bar.

trations are reached substantially faster. Below 8 bar, the systematic error due to the neglected $\mathrm{OH}$ superequilibrium exceeds the above mentioned $60 \mathrm{~K}$ inherent systematic uncertainty due to the residual equivalence ratio dependence of the $\mathrm{OH}$ concentration in the lean regime. Therefore, at pressures below approximately 8 bar, no physical temperature values can be deduced from $\mathrm{OH}$ concentration in the flame front.

Figure 12 shows LIF- and CARS-based axial temperature profiles for different equivalence ratios. Here, the issue of applicability of the $\mathrm{OH}$ absorption/fluorescence technique at different equivalence ratios, and the reliability of temperature measurements near or even above the theoretical limit of $\Phi=0.9$, as discussed above, is addressed. The decrease of temperatures with equivalence ratio is clearly visible. Again, there is very good agreement between the result of the two methods; however, in the case of a fuel-rich flame $(\Phi=1.1)$, the agreement is good only if the equivalence ratio is known, and the dependence of the measured $\mathrm{OH}$ concentration on both temperature and equivalence ratio is accounted for. If the stoichiometry dependence of the temperature is neglected and a fuel-lean flame is assumed (as would be the case in a turbulent flame in a gas turbine combustor with a lean global equivalence ratio and no method to determine the local and instantaneous equivalence ratio simultaneously), the resulting temperature would be about $300 \mathrm{~K}$ too low, indicated by the dashed curve labelled "T $[\mathrm{OH}]$ lean, $\Phi=1.1$ ". This underlines the importance of the prerequisite of a lean mixture everywhere, not only globally, for the applicability of the OH LIF/absorption thermometry method.

The flames with different equivalence ratios and temperatures provide the possibility to prove the assumptions of a constant fluorescence quantum yield, which is the fraction of $\mathrm{OH}$ radicals excited by the laser which actually emits fluorescence, rather than loosing the excitation energy by radiationless collisions. This issue is of no importance in these extremely homogeneous flames. But in turbulent flames a fluorescence quantum yield along the laser beam path through the flame will vary from the local values which are iteratively approximated using relative values calculated by the LASKIN code (see Fig. 2 and Table 1). A changing fluorescence quantum yield 
would manifest itself in a changing ratio of the integral absorption $a_{0}$ and the fluorescence intensity integrated along the laser beam path through the flame. In the presented experiment we determined the ratio $a_{0}$ of integral absorption to the integral fluorescence for the quite homogeneous flames with $\Phi=0.75$ and $\Phi=1.1$. Fortunately, the $\mathrm{OH}$ concentrations of these flames were comparable, so that the influence of fluorescence reabsorption on the ratio of the quantum yields would cancel. The ratio $a_{0}(\Phi=1.1) / a_{0}(\Phi=0.75)=1.19 \pm 0.07$ is in agreement with the calculated values listed in Table 1.

\subsection{Application to an LPP injector investigation at elevated pressures}

Lean premixed prevaporized (LPP) combustion is a promising approach to reduce emissions of nitric oxides $\left(\mathrm{NO}_{x}\right)$. By prevaporizing the liquid fuel and by homogenizing the lean fuel air mixture inside the burner prior to combustion, high temperatures resulting from near stoichiometric combustion can be avoided [29]; at the same time, this concept fulfils the basic requirement for the application of the PLIF/absorption thermometry strategy, namely a lean mixture prior to combustion.

While being well established for gas turbines for power generation running on gaseous fuels, LPP injectors for aero engines mean considerable larger technical challenges, due to the higher load range and the additional complexity associated with a liquid fuel, as well as safety and operability concerns.

An LPP injector with a new concept [14] was developed at the DLR Institute of Propulsion Technology. The basic idea behind the concept is to spatially separate atomization and homogenization inside the injector, allowing individual optimization of these processes.

The injector was operated in a Single Sector Combustor (SSC) with optical access from three sides to the primary zone. For details of the test rig, the LPP injector and operating conditions, the reader is referred to [14].

The operating conditions are characterized by the combustor pressure $p$, the air preheat temperature $T_{3}$ and equivalence ratio $\Phi_{\mathrm{pz}}$ calculated from the primary zone. As the primary zone has film cooled windows, the contribution of the film cooling air $(27 \%)$ is included in the calculation of $\Phi_{\mathrm{pz}}$.

For the planar optical measurement techniques, especially the simultaneous kerosene/OH PLIF and absorption measurement, the optical setup shown in Fig. 3 was used. In contrast to the OH-PLIF validation measurements in a methane/air flame, in a kerosene/air flame the OH PLIF signal spectrally interferes with kerosene PLIF. Therefore the kerosene PLIF has to be measured by an additional camera recording a different spectral range (see Fig. 4) to separate the $\mathrm{OH}$ and kerosene LIF signals by a linear combination of the images of camera 1 and camera 2. An exemplary result of the combined kerosene/OH PLIF and absorption measurement on a single-pulse basis is shown in Fig. 13. The images were taken at an equivalence ratio of $\Phi_{\mathrm{pz}}=0.42$ referring to the total primary zone air, or $\Phi_{\text {injector }}=0.53$ with respect to injector air flow only. The laser light sheet starts $5 \mathrm{~mm}$ above the burner exit plane and has a height of $48 \mathrm{~mm}$.

Despite the low residence time of $\tau=0.59 \mathrm{~ms}$ in the burner almost all of the kerosene is evaporated at an air preheat temperature of $T_{3}=850 \mathrm{~K}$, which could be confirmed by images of the Mie scattering from droplets and by Phase Doppler 

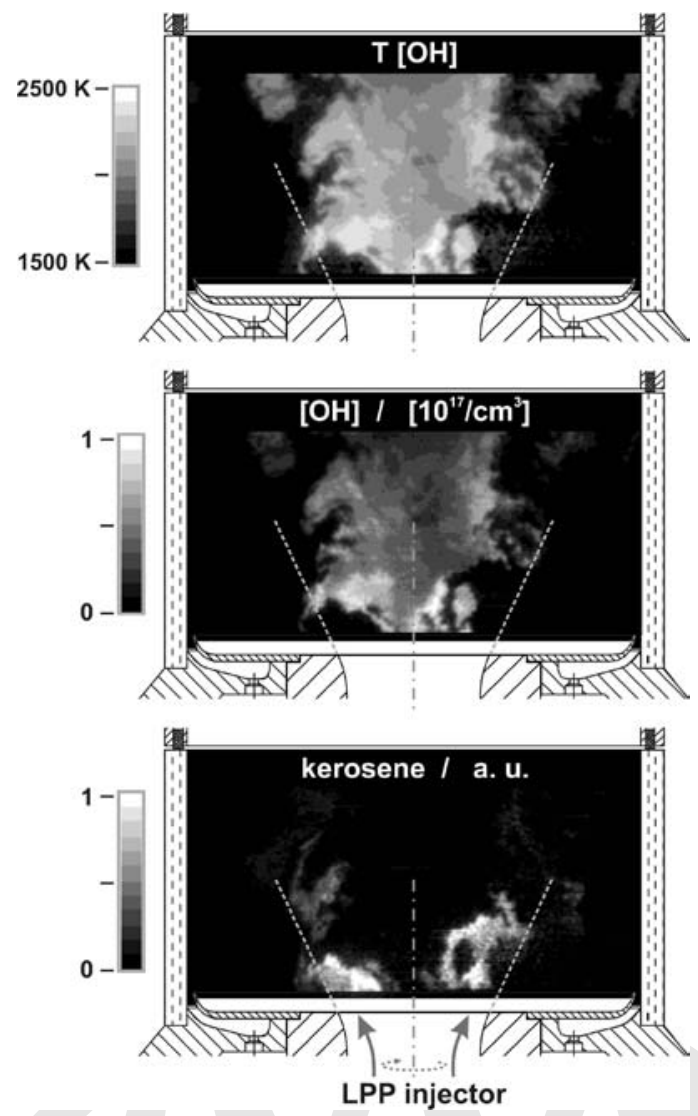

Fig. 13. Vaporized kerosene (bottom) and $\mathrm{OH}$ distribution (middle) of the LPP injector flame at $\Phi_{\text {injector }}=$ $0.53, T_{3}=850 \mathrm{~K}, \mathrm{p}_{3}=6 \mathrm{bar}$, deduced from a simultaneous kerosene and OH PLIF and absorption measurement on a single-shot base. The $\mathrm{OH}$ concentration is converted to the temperature distribution (top).

Anemometry measurements, see [30]. The "kerosene" PLIF images are qualitative in the sense that they show strictly only the distribution of the aromatics in the combustor that survived thermal decomposition. Nevertheless, the complementary character of the "fuel" and the hot exhaust gas distribution is impressive. Near the boundary between fuel (bottom image) and the $\mathrm{OH}$ concentration (middle image), no well defined margin of the $\mathrm{OH}$ superequilibrium of the flame front is visible. As mentioned in the discussion of the method, the authors observed such $\mathrm{OH}$ superequilibrium flame front structures only in fuel-rich turbulent pressurized flames. Assuming a locally lean flame, the $\mathrm{OH}$ concentration distribution can be converted to the temperature field shown in the top of Fig. 13. The noise level of these temperature images is low enough for single pulse analysis, due to the high line strength of the chosen $\mathrm{OH}$ transition. The exponential growth of the $\mathrm{OH}$ equilibrium concentration with respect to temperature leads to a lower temperature limit of about $1500 \mathrm{~K}$. The $\mathrm{OH}$ detection limit in kerosene/air flames is significantly determined by the limited correction for kerosene 

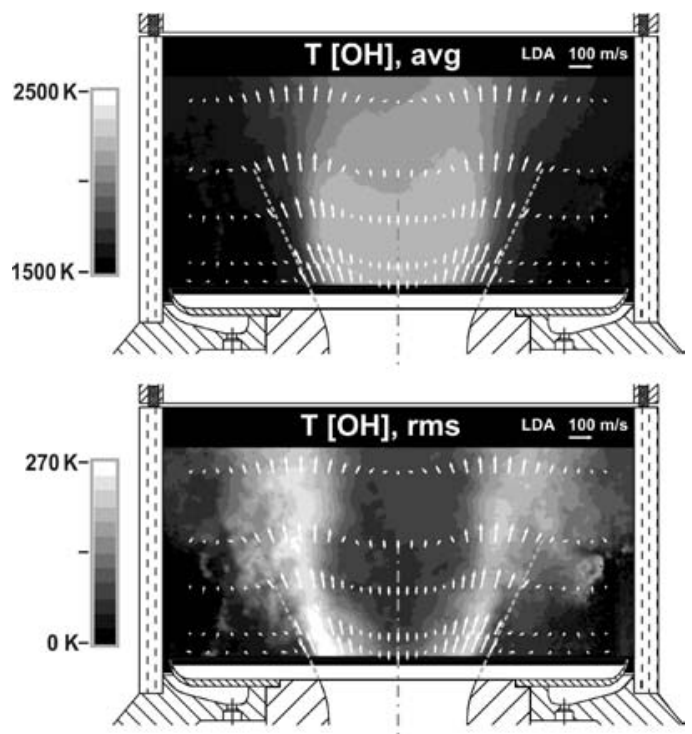

Fig. 14. Averaged temperature (top) and the RMS temperature (bottom) distributions of 100 single-shot measurements. Additionally the velocity vectors of the LDA measurement are superimposed.

LIF interferences. Therefore, only temperature values above $1500 \mathrm{~K}$ were taken into account to calculate the mean temperature field. The averaged temperature field and the RMS values of 100 single-pulse measurements are shown in Fig. 14. The velocity field measured by Laser Doppler Anemometry (LDA) is superimposed on both images. The highest temperature fluctuations are found in the region of the highest velocities of the injector. The inner recirculation zone shows a stable temperature distribution with a low fluctuation of $T_{\mathrm{RMS}} \leq 65 \mathrm{~K}$. At the burner exit temperatures in the inner recirculation zone are measured that exceed the adiabatic flame temperature of $2030 \mathrm{~K}$ for this flame by about $200 \mathrm{~K}$, indicating a still incomplete mixing of fuel and air as they leave the injector. At the end of the inner recirculation zone $50 \mathrm{~mm}$ above the burner exit plane the temperature reaches the adiabatic flame temperature.

In general "fluorescence trapping", i.e., the reabsorption of the OH LIF by OH itself or by other species on the way to the detector, can be a serious problem to quantify the LIF signal. Especially in an environment of high $\mathrm{OH}$ concentrations, $\mathrm{OH}$ fluorescence trapping is an issue [31]. To reduce the influence of $\mathrm{OH}$ fluorescence trapping, the $\mathrm{OH}$ camera is equipped with an additional long-pass filter (SCHOTT WG305). It attenuates the fluorescence of the $A-X(0,0)$ band at $305 \mathrm{~nm}$, which is very sensitive for reabsorption due to the high thermal population of the absorbing states, more than the fluorescence of the $A-X(1,1)$ band at $315 \mathrm{~nm}$.

But the first and most important step to correct for the influence the fluorescence trapping is done by the normalization the LIF to the ratio of the integral absorption and the integral LIF, $a_{0}$, as described in Eq. (7). By this way, at least the integral $[\mathrm{OH}]$ value is correct, while the local $[\mathrm{OH}]$ values are approximated. 
To examine the influence of the fluorescence trapping by $\mathrm{OH}$ itself in more detail, we compared the $a_{0}$ values of flames with different $\mathrm{OH}$ concentrations. By varying $\Phi_{\text {injector }}$ from 0.53 to $0.66\left(T_{3}=850 \mathrm{~K}, p=6 \mathrm{bar}\right)$, the $\mathrm{OH}$ concentration increases by $85 \%$, while the $a_{0}$ value increases by $11 \%$. The correlated change of the fluorescence quantum yield (Table 1) would explain $9 \%$ of the $11 \%$ change of $a_{0}$. Thus, the influence of fluorescence trapping in our experiment is measureable, but leads not to serious systematic errors.

\section{Summary}

The concept of planar temperature measurements based on $\mathrm{OH}$ concentrations, which are derived from simultaneous measurements of laser-induced fluorescence and absorption, was tested by comparison with SV-CARS flame temperature measurements.

The experiments were performed in several premixed, laminar $\mathrm{CH}_{4} /$ air flames at elevated pressure; these flames allowed independent control of the key parameters pressure and equivalence ratio. According to theoretical considerations, the applicability of the method is limited to equivalence ratios of 0.9 or less; therefore, an assessment of the reliability of temperature measurements at different equivalence ratios, specifically near or even above this threshold, was one aim of this study. Temperature measurements were performed at different stoichiometries. An excellent agreement of the $\mathrm{OH}$ PLIF-based temperatures using both single pulse, i.e., time-resolved measurements, and average images with temperatures measured by CARS was found at all pressures and equivalence ratios. In particular, the good single pulse capability was demonstrated. The conjecture resulting from theoretical considerations, namely, that the method will yield reliable temperatures without knowledge of the equivalence ratio only if the flame is fuel-lean everywhere, was confirmed; even in only slightly fuel-rich flames, the strong dependence of $\mathrm{OH}$ equilibrium concentrations on equivalence ratio leads to errors of more than 100 degrees if the equivalence ratio is not known and assumed to be lean.

The fact that the method is based on the measurement of equilibrium $\mathrm{OH}$ concentrations implies a further restriction of its applicability. It was shown that in $\mathrm{CH}_{4} /$ air flames below approximately $6 \mathrm{bar}$, the superequilibrium concentrations in flame front regions resulting from slower relaxation at lower pressures result in erroneous temperatures.

In summary, the method has been demonstrated to be applicable under conditions prevailing in lean burn gas turbine combustors, namely lean mixtures everywhere, and pressures above approximately 6 bar. In addition, full optical access to the entire flame is required, in order to relate an integral absorption measurement with a PLIF signal. Under these conditions, the remaining small variation of the $\mathrm{OH}$ equilibrium concentration with stoichiometry in the lean regime leads to a systematic temperature error of about $60 \mathrm{~K}$. A general statement on the accuracy of the method is difficult to give, because the statistical errors are strongly temperature-dependent, due to the almost exponential dependence of the $\mathrm{OH}$ concentration and the associated LIF signal on temperature. For the same reason, the accessible temperature range is limited to values above approximately $1400 \mathrm{~K}$, depending on optical access and pressure. 


\section{Acknowledgement}

This work received funding from the European Community through the $6^{\text {th }}$ Framework Programme Aeronautics \& Space, project TLC - Towards Lean Combustion (Contract \# 012326). The financial support of the German Ministry for Economy and Labor under Förderkennzeichen AG TurboII 0327090E is gratefully acknowledged. It reflects only the author's views and the Community is not liable for any use that may be made of the information contained therein.

\section{References}

1. W. Stricker, R. Lückerath, U. Meier, and W. Meier, J. Raman Spectrosc. 34 (2003) 922.

2. S. Kampmann, T. Seeger, and A. Leipertz, Appl. Opt. 34 (1995) 2780.

3. L. Wehr, W. Meier, P. Kutne, and C. Hassa, Proc. Combust. Inst. (2007) 3099.

4. J. Seitzman and R. K. Hanson, Appl. Phys. B 57 (1993) 385.

5. R. Giezendanner-Thoben, U. Meier, W. Meier, and M. Aigner, Flow, Turbulence Combust. 75 (2005) 317.

6. R. Devillers, G. Bruneaux, and C. Schulz, Appl. Opt. 47 (2008) 5871.

7. A. O. Vyrodov, J. Heinze, M. Dillmann, U. E. Meier, and W. Stricker, Appl. Phys. B 61 (1995) 409.

8. M. P. Lee, B. K. McMillin, and R. K. Hanson, Appl. Opt. 32 (1993) 5379.

9. J. Hult, I. S. Burns, and C. F. Kaminski, Proc. Combust. Inst. 30 (2005) 1535.

10. S. M. Correa, Combust. Sci. Tech. 87 (1992) 329.

11. A. Arnold, R. Bombach, B. Käppeli, and A. Schlegel, Appl. Phys. B 64 (1997) 579.

12. M. Braun-Unkhoff, DLR Stuttgart, Institute of Combustion Technology, private communication (2006).

13. C. Morley, "GASEQ - A Chemical Equilibrium Program for Windows", http://www.gaseq. co.uk/.

14. T. Behrendt, J. Heinze, and C. Hassa, Proc. of ASME Turbo Expo (2003) GT2003-38444, ISBN 0-7918-3671-1.

15. R. Giezendanner-Thoben, U. Meier, W. Meier, J. Heinze, and M. Aigner, Appl. Opt. 44 (2005) 6565 .

16. W. Demtröder, Laser Spectroscopy, Springer Series in Chemical Physics 5, Springer Berlin, Heidelberg (1981).

17. G. Dieke and H. Crosswhite, J. Quant. Spectrosc. Radiat. Transfer 2 (1962) 97.

18. U. Rahmann, A. Bülter, U. Lenhard, R. Düsing, D. Markus, A. Brockhinke, and K. KohseHöinghaus, LASKIN - A Simulation Program for Time-Resolved LIF-Spectra, Internal Report, University of Bielefeld, Faculty of Chemistry, Physical Chemistry I, http://pc1.unibielefeld.de/ laskin.

19. R. Trolier, Kinetic and Spectroscopic Studies of Ozone Photochemistry, Ph.D. Thesis, Cornell University, (1988).

20. J. Luque and D. Crosley, J. Chem. Phys. 109 (1998) 439.

21. A. C. Eckbreth, Laser Diagnostics for Combustion Temperature and Species, Eds. A. K. Gupta and D. G. Lilley, Abacus Press, ISBN 085626 3443, pp. 82ff.

22. U. E. Meier, D. Wolff-Gassman, and W. Stricker, Aerosp. Sci. Technol. 4 (2000) 403.

23. M. Tsurikov, K. P. Geigle, Y. Schneider-Kühnle, W. Stricker, R. Lückerath, R. Hadef, and M. Aigner, Combust. Sci. Technol. 177 (2005) 1835.

24. K. P. Geigle, Y. Schneider-Kühnle, M. Tsurikov, R. Hadef, R. Lückerath, V. Krüger, W. Stricker, and M. Aigner, Proc. Combust. Inst. 30 (2005) 1645.

25. R. Lückerath, M. Woyde, W. Meier, W. Stricker, U. Schnell, H. Magel, J. Görres, H. Spliethoff, and H. Maier, Appl. Opt. 34 (1995) 3303.

26. J. A. Coxon, Can. J. Phys. 58 (1980) 933.

27. R. Schließ1, P. Pixner, A. Dreizler, and U. Maas, Combust. Sci. Tech. 149 (1999) 339. 
28. B. Atakan, J. Heinze, and U. E. Meier, Appl. Phys. B 64 (1997) 585.

29. V. J. Lyons, AIAA-81-0327, 1981.

30. T. Behrendt, Strömung und Verbrennung in einem neuen Düsenkonzept für die magere Vormischverbrennung, PhD Thesis, University Bochum (2003) ISBN 1434-8454.

31. R. Sadanandan, W. Meier, and J. Heinze, Appl Phys. B, online first (2011), DOI: 10.1007/ s00340-011-4704-z. 